NOVA

University of Newcastle Research Online

nova.newcastle.edu.au

Ratnam, Elizabeth L.; Weller, Steven R.; Kellett, Christopher M. "An optimization-based approach to scheduling residential battery storage with solar PV: assessing customer benefit" Renewable Energy Vol. 75, p. 123-134 (2015)

Available from: http://dx.doi.org/10.1016/j.renene.2014.09.008

Accessed from: http://hdl.handle.net/1959.13/1056697 


\title{
An optimization-based approach to scheduling residential battery storage with solar PV: Assessing customer benefit
}

\author{
Elizabeth L Ratnam $^{\mathrm{a}, 1, *}$, Steven R. Weller ${ }^{\mathrm{a}, 1}$, Christopher M. Kellett ${ }^{\mathrm{a}, 1}$ \\ ${ }^{a}$ The University of Newcastle, School of Electrical Engineering and Computer Science, University Drive, Callaghan NSW 2308, Australia
}

\begin{abstract}
Several studies have suggested that battery storage co-located with solar photovoltaics (PV) benefits electricity distributors in maintaining system voltages within acceptable limits. However, without careful coordination, these potential benefits might not be realized. In this paper we propose an optimization-based algorithm for the scheduling of residential battery storage co-located with solar PV, in the context of PV incentives such as feed-in tariffs. Our objective is to maximize the daily operational savings that accrue to customers, while penalizing large voltage swings stemming from reverse power flow and peak load. To achieve this objective we present a quadratic program (QP)-based algorithm. To complete our assessment of the customer benefit, the QP-based scheduling algorithm is applied to measured load and generation data from 145 residential customers located in an Australian distribution network. The results of this case study confirm the QP-based scheduling algorithm significantly penalizes reverse power flow and peak loads corresponding to peak time-of-use billing. In the context of feed-in tariffs, the majority of customers exhibited operational savings when QP energy-shifting.
\end{abstract}

Keywords: Photovoltaics, battery scheduling, feed-in tariffs, time-of-use pricing, peak-load reduction.

\section{Introduction}

Climate change, energy security, and limited fossil fuel resources are drivers for the integration of renewable energy sources such as solar into the modern power grid. Significant challenges in converting the abundant solar resource into reliable, high-quality electricity include variability of solar irradiance on both daily and seasonal timescales in addition to intermittency arising from moving cloud cover on timescales of much shorter duration [1,2].

Despite these challenges, governments around the world have in recent years encouraged grid-integrated residentialscale (rooftop) solar photovoltaic (PV) generation through financial incentives such as feed-in-tariffs (FiTs) paid directly to customers [3-5]. These financial incentives in conjunction with a sharp drop in the capital cost of smallscale PV, and increasing electricity prices, have led to the dramatic uptake of residential PV in some countries [6, 7]. For example, in Germany PV plant installations exceed 1.2 million, and as of September 2012, peak PV capacity reached $31 \mathrm{GW}$ with about $70 \%$ of this $31 \mathrm{GW}$ being connected to the low voltage grid [6].

An adverse consequence of such significant PV penetration in the low voltage electricity distribution network is voltage rise leading to reverse power flow. Voltage rise is particularly pronounced when large numbers of rooftop PV generators are connected in close proximity to each other [8-14]. A further adverse consequence of significant PV penetration is voltage dip. This occurs, for example, when passing cloud cover results in a significant drop in rooftop

\footnotetext{
${ }^{*}$ Corresponding author

Email addresses: elizabeth.ratnam@ieee.org (Elizabeth L Ratnam), Steven.Weller@newcastle.edu .au (Steven R. Weller ), Chris.Kellett@newcastle.edu.au (Christopher M. Kellett )

${ }^{1}$ Corresponding author: Elizabeth L Ratnam, Tel.: +61 2492 16026, Fax: +61 249216993.
} 
PV generation $[10-12,15]$. If these voltage deviations fall outside power quality standards, either the utility covers the direct cost of mitigation or the burden of voltage regulation falls to the PV producer [2, 6, 8, 13, 14].

There are two common approaches to managing voltage rise in the low voltage grid. The first is to augment the distribution grid by increasing conductor size and/or upgrading transformers to lower network impedances $[6,9,16]$. The second is to constrain PV generation at times of low electricity consumption in order to preserve compliance of allowable voltage deviations $[13,17,18]$. Neither approach is optimal for increased PV penetration as network augmentation adds to the overall PV grid integration costs [9] whereas spilling PV generation leads to lost revenue for the producer.

Alternative approaches to managing PV generation in the low voltage grid are facilitated through Advanced Metering Infrastructure (AMI) [19-23]. When two way communication is enabled between the utility and customer via AMI, opportunities exist for more advanced demand-side management initiatives that include direct [20, 24-28], and price-responsive $[21-23,29,30]$ load control. For example, the utility can enact price-responsive load control by broadcasting a day-ahead time-varying electricity tariff to the AMI. To maintain an existing energy usage level, the customer may choose to schedule battery storage in response to the time-varying electricity tariff or pay a higher energy bill. However without careful coordination of the residential battery schedules, network load curve smoothing via demand-side management initiatives may not be realized [26, 31, 32].

Several authors have investigated energy-time shifting through battery storage with a focus on minimizing residential energy bills and reducing network peak demand [33-37], leading to battery schedules that either assist or exacerbate non-compliant voltage deviations associated with solar PV. The reduction of network peak demand is incorporated into an optimization problem in [34], where the objective function includes financial incentives for residents to deliver energy to the grid when the purchase cost of electricity is high. Hence, when interconnected customers in close proximity implement the objective function in [34], large voltage swings associated with reverse power flow potentially arise due to the battery scheduling. The reduction of network peak demand is also incorporated into a linear program in [33], where the energy flowing from the point of common coupling (PCC) to the customer is minimized when residential load exceeds residential PV production. Otherwise the battery is scheduled in [33] to charge during the off-peak pricing period, and discharge during the peak pricing period, with no penalty on increased reverse power flow, potentially exacerbating voltage rise. In contrast, the reduction of network peak demand and the mitigation of undesirable reverse power flow, i.e., load curve smoothing, is incorporated into the optimization problems in [3537]. The optimization problem in [35] achieves load curve smoothing by omitting financial incentives encouraging solar PV uptake (e.g., feed in tariffs or net metering) in the objective function. The optimization problem in [36] also achieves load curve smoothing by removing incentives for reverse power flow associated with battery scheduling, while permitting incentives encouraging solar PV uptake. Another method for reducing network peak demand while potentially abating reverse power flow is incorporated into the optimization problem in [37], where a sophisticated dynamic pricing environment provides additional incentives for customers to smooth their day-ahead energy consumption.

Our objective in this paper is similar to [36] in one respect, we seek to maximize residential PV generation co-located with battery storage so that there is a financial benefit to the resident whilst simultaneously alleviating the utility burden associated with peak demand and reverse power flow. Our approach achieves this objective for a range of financial incentives offered for solar PV uptake, such as feed-in tariffs [3-5, 36] and net metering [34, 38, 39], in addition to other more sophisticated dynamic day-ahead pricing rates [23, 29, 37]. We assume peak billing rates coincide with generation shortages or peak grid demand and look to minimize energy flow from the grid to the customer during these events, while additionally reducing reverse power flow.

Implicit in our approach is the expectation that residential customers have installed Home Energy Management (HEM) systems that: (1) forecast the day-ahead residential load and solar PV generation, (2) coordinate with the AMI to receive day-ahead prices for energy delivered to and from the grid, including any additional PV incentives, (3) run optimization-based algorithms daily, and (4) schedule battery storage in the day-ahead. In this paper we assume the day-ahead forecast of load and generation from the HEM system are known and perfect, and we focus on the formulation of an optimization-based algorithm that provides the day-ahead battery schedule. We also assume the customers' HEM system is fully automated and employs a wireless communication architecture, similar to the description in 
[27]. Furthermore, we expect global investment and government mandates will drive both technology improvements and economies of scale for battery storage as has happened with solar PV [40-43]. Therefore our focus is on the operational savings that accrue to a resident when the HEM schedules a battery, and we exclude the capital costs of purchasing a battery from our consideration.

In this paper we consider the quadratic program (QP)-based minimization of the energy supplied by, or to, the grid in a residential PV system with co-located battery storage, first presented in [44]. Our objective is to smooth network load curves while providing incentives to customers to energy time-shift. In the present paper we remove a bias in the QP-based algorithm in [44] by including an additional battery constraint related to the state of charge, and with a modification to the greedy-search heuristic that selects the key design parameters in this QP we reduce computational time. Furthermore, we apply the improved QP-based scheduling algorithm to measured load and generation data from 145 Australian residential customers, and investigate the financial savings that accrue to customers. In the present paper, the financial benefit associated with the daily battery charge/discharge schedule is our primary focus in the context of financial incentives offered for solar PV generation such as feed-in tariffs, rather than the utility benefit of load curve smoothing.

This paper is organized as follows. In Section 2 we introduce the optimization-based approach for scheduling battery storage in a residential PV system, and include a motivating example. To assess the customer benefit, we introduce a framework in Section 3 that incorporates different demand-side management approaches for price-response load control, which integrates applicable incentives for PV generation. With this framework we define the daily energy bill for a single customer with and without battery storage. In Section 4 we describe the operations savings associated with battery scheduling, and in Section 5 we present an algorithm for selecting a key design parameter in the QP described in Section 2. In Section 6 we implement the QP-based algorithm given real-world data from 145 residential customers located in an Australian distribution network in the context of feed-in tariffs, and investigate the customer benefit to changes in different elements within the QP (e.g., battery size).

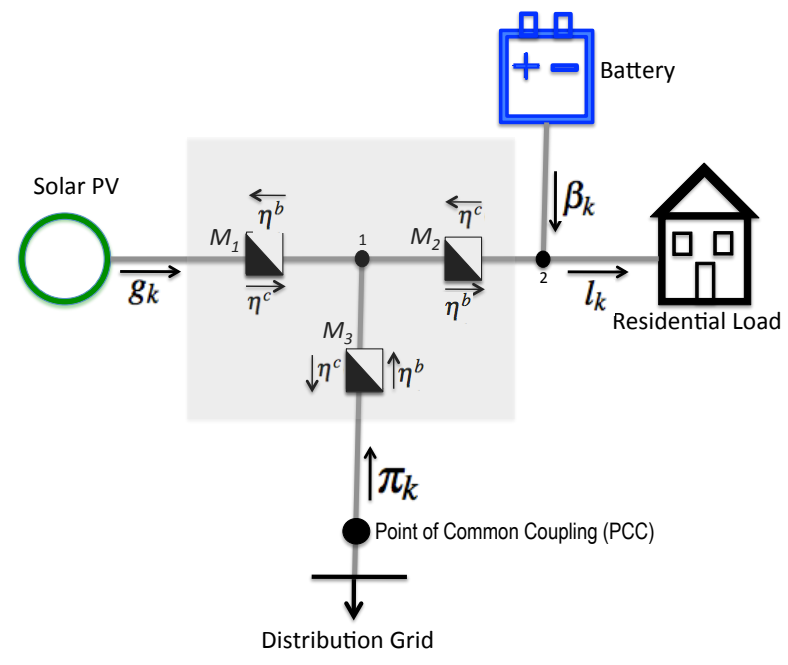

Figure 1: Residential system illustrating the direction of positive power flows and financial incentives to energy time-shift. Arrows associated with $g_{k}, l_{k}, \beta_{k}$ and $\pi_{k}$ illustrate the assumed direction of positive power flow. Financial incentives for each meter $M_{1}, M_{2}$ and $M_{3}$ are represented by vectors $\eta^{b}$ and $\eta^{c}$ (in $\$ / \mathrm{kWh}$ ), in which arrows illustrate the direction of power flow relevant for $\eta^{b}$ and $\eta^{c}$.

\section{Notation}

Let $\mathbb{R}^{s}$ denote $s$-dimensional vectors of real numbers and $\mathbb{R}_{\geq 0}^{s} s$-dimensional vectors with all non-negative components where, as usual, $\mathbb{R}^{1}=\mathbb{R}$. I denotes the $s$-by-s identity matrix and $\mathbb{1} \in \mathbb{R}_{\geq 0}^{s}$ denotes the all-1s column vector of length $s$. $\mathbf{0}$ denotes an all-zero matrix, or an all-zero column vector, where the context will make clear the dimension intended, and $\mathbf{T}=\left[t_{i j}\right]$ denotes the $s$-by-s matrix satisfying $t_{i j}=1$ for $i \geq j$ and $t_{i j}=0$ elsewhere. 


\section{Problem formulation}

\subsection{Definitions and constraints}

Figure 1 illustrates the topology of the system under consideration, including a set of meters $\mathscr{M}=\left\{M_{1}, M_{2}, M_{3}\right\}$ installed for the purpose of billing and compensation. For each $k \in\{1, \ldots, s\}$, meter $M_{1}$ measures the average PV generation $g_{k}$ (in $\mathrm{kW}$ ), meter $M_{2}$ measures the average power from node 1 to node $2\left(l_{k}-\beta_{k}\right.$ in $\mathrm{kW}$ ), and meter $M_{3}$ measures the average power $\pi_{k}$ (in $\mathrm{kW}$ ) supplied by (or to) the grid. Meters $M_{2}$ and $M_{3}$ may be bi-directional, whereas meter $M_{1}$ needs only be unidirectional since PV generation satisfies $g_{k} \geq 0$ for all $k$. Also shown in Figure 1 are vectors $\eta^{b}$ and $\eta^{c}$, which represent financial incentives for billing and compensation respectively, defined in Section 3.2.

The power flows indicated in Figure 1 are represented by vectors of length $s$, where $s$ is the number of time intervals of length $\Delta$, and $T=s \Delta$ (in hours) is the time window of interest. In this paper we generally consider $T=24$ hours, $\Delta=1 / 2$ hour (30 minutes), which implies $s=48$. Other choices are certainly possible, subject only to commensurability of $T, \Delta$ and $s$.

We represent the average power delivered to the residential load (in $\mathrm{kW}$ ) over the period $((k-1) \Delta, k \Delta)$ by $l_{k}$ for all $k \in$ $\{1, \ldots, s\}$, and define the load profile over $[0, T]$ as $l:=\left[l_{1}, \ldots, l_{s}\right]^{T} \in \mathbb{R}_{\geq 0}^{s}$. Likewise we represent the average PV generation $(\mathrm{kW})$ over the period $((k-1) \Delta, k \Delta)$ by $g_{k}$ for all $k \in\{1, \ldots, s\}$, and define the generation profile over $[0, T]$ as $g:=\left[g_{1}, \ldots, g_{s}\right]^{T} \in \mathbb{R}_{>0}^{s}$. In what follows, we assume the day-ahead forecasts of load and generation profiles are known and perfect.

We represent the average power (in $\mathrm{kW}$ ) supplied by (or to) the grid over the period $((k-1) \Delta, k \Delta)$ by $\pi_{k}$ for all $k \in\{1, \ldots, s\}$ and define the grid profile over $[0, T]$ as $\pi:=\left[\pi_{1}, \ldots, \pi_{s}\right]^{T} \in \mathbb{R}^{s}$. By convention we represent power flowing from (to) the grid to (from) the energy system by $\pi_{k}>0\left(\pi_{k}<0\right)$.

We represent the average power ( $\mathrm{kW}$ ) delivered from (or to) the battery over the period $((k-1) \Delta, k \Delta)$ by $\beta_{k}>0$ (or $\left.\beta_{k}<0\right)$ for all $k \in\{1, \ldots, s\}$, and define the battery profile over $[0, T]$ as $\beta:=\left[\beta_{1}, \ldots, \beta_{s}\right]^{T} \in \mathbb{R}^{s}$. By convention we represent charging (discharging) of the battery by $\beta_{k}<0\left(\beta_{k}>0\right)$.

From the configuration of the residential energy system in Figure 1, we observe that the following power balance equation

$$
l_{k}=\pi_{k}+g_{k}+\beta_{k} \quad \text { for all } k \in\{1, \ldots, s\},
$$

must hold.

The inclusion of the battery in Figure 1 leads to additional constraints, which we now detail. To capture the limited "charging/discharging capacity" of the battery, we constrain $\beta$ by

$$
\underline{B} \mathbb{1} \leq \beta \leq \bar{B} \mathbb{1}
$$

where $\underline{B} \in \mathbb{R}_{\leq 0}$ and $\bar{B} \in \mathbb{R}_{\geq 0}$.

Given $\beta$, the state of charge of the battery (in $\mathrm{kWh}$ ) at time $k \Delta$ is denoted by $\chi_{k}$, where

$$
\chi_{k}:=\chi_{0}-\sum_{j=1}^{k} \beta_{j} \Delta \quad \text { for all } k \in\{1, \ldots, s\},
$$

and $\chi_{0}$ denotes the initial state of charge of the battery. We represent the state of charge profile by $\chi:=\left[\chi_{0}, \ldots, \chi_{s}\right]^{T} \in$ $\mathbb{R}^{s+1}$.

If we represent the battery capacity (in $\mathrm{kWh}$ ) by $C \in \mathbb{R}_{\geq 0}$, it necessarily follows that the state of charge profile is constrained by

$$
0 \leq \chi \leq C\left[\begin{array}{l}
1 \\
\mathbb{1}
\end{array}\right]
$$


For a fixed initial state of charge satisfying $0 \leq \chi_{0} \leq C$, we define $\underline{\mathbf{C}}:=\left(\chi_{0} / \Delta\right) \mathbb{1}$, and $\overline{\mathbf{C}}:=(1 / \Delta)\left(C-\chi_{0}\right) \mathbb{1}$, and rewrite the battery constraints (3)-(4) as

$$
-\underline{\mathbf{C}} \leq-\mathbf{T} \beta \leq \overline{\mathbf{C}} .
$$

In this paper, we optimize a battery profile over a single day. In order to avoid an energy-shifting bias in these results, we insist that the state of charge of the battery at the end of a day is the same as the state of charge of the battery at the beginning of the day, i.e.,

$$
\chi_{s}=\chi_{0}
$$

where $\chi_{s}$ is the final state of charge at time $s \Delta$.

Let $A_{1} \in \mathbb{R}^{4 s \times s}$, and $b_{1} \in \mathbb{R}^{4 s}$ be defined by

$$
A_{1}:=\left[\begin{array}{llll}
\mathbf{I} & -\mathbf{I} & \mathbf{T} & -\mathbf{T}
\end{array}\right]^{T}, \quad \quad b_{1}:=\left[\begin{array}{llll}
\bar{B} \mathbb{1}^{T} & \underline{B} \mathbb{1}^{T} & \underline{\mathbf{C}}^{T} & \overline{\mathbf{C}}^{T}
\end{array}\right]^{T} .
$$

We now substitute equations (7) into equations (2) and (5), and equation (6) into (3), to succinctly write the battery constraints as

$$
\begin{aligned}
& A_{1} \beta \leq b_{1}, \\
& \mathbb{1}^{T} \beta=0 .
\end{aligned}
$$

\subsection{Objectives}

In what follows, we seek to minimize the impact of the residential energy system on the grid, given a financial incentive to energy time-shift, by minimizing

$$
\sum_{k=1}^{s} h_{k} \pi_{k}^{2}
$$

where $h_{k}$ is a selectable weighting such that $h_{k} \geq 1$ for all $k \in\{1, \ldots, s\}$.

Specifically, given load and generation profiles $l$ and $g$, and given battery constraints $\chi_{0}, C$, and $\bar{B}, \underline{B}$ we seek a battery profile $\beta$ and a grid profile $\pi$ which minimize the expression in (10), subject to satisfaction of the power balance in equation (1).

The minimization in (10) is subject to both inequality and equality constraints imposed by the battery (8)-(9) and the power balance equation in (1), respectively. Lemma 1 below establishes this constrained minimization as a quadratic program (QP).

Lemma 1. The minimization of expression (10), subject to battery constraints (8)-(9) and the power balance equation (1), can be written as

$$
\min _{x \in \mathbb{R}^{2 s}} x^{T} H x
$$

such that

$$
\begin{aligned}
& \bar{A}_{1} x \leq b_{1}, \\
& A_{2} x=b_{2},
\end{aligned}
$$

where

$$
\begin{aligned}
& x:=\left[\begin{array}{ll}
\pi^{T} & \beta^{T}
\end{array}\right]^{T} \quad \in \mathbb{R}^{2 s}, \\
& H:=\left[\begin{array}{cc}
\mathbf{H} & \mathbf{0} \\
\mathbf{0} & \mathbf{0}
\end{array}\right] \quad \in \mathbb{R}^{2 s \times 2 s}, \\
& \mathbf{H}:=\operatorname{diag}\left(h_{1}, \ldots, h_{s}\right) \quad \in \mathbb{R}^{s \times s}, \\
& \bar{A}_{1}:=\left[\begin{array}{ll}
\mathbf{0} & A_{1}
\end{array}\right] \quad \in \mathbb{R}^{4 s \times 2 s}, \\
& A_{2}:=\left[\begin{array}{cc}
\mathbf{0}^{T} & \mathbb{1}^{T} \\
\mathbf{I} & \mathbf{I}
\end{array}\right] \quad \in \mathbb{R}^{(s+1) \times 2 s}, \\
& b_{2}:=\left[\begin{array}{c}
0 \\
l-g
\end{array}\right] \quad \in \mathbb{R}^{s+1} \text {. }
\end{aligned}
$$


Proof. The result follows directly from equations (1), (8), and (9).

The grid profile obtained by solving (11) subject to constraints (12)-(13) is said to be QP energy-shifted and we will refer to the process of a customer implementing the daily battery and grid profiles obtained by solving (11) subject to constraints (12)-(13) as QP energy-shifting.

\subsection{Example}

In this example we consider two battery capacities, $C=1 \mathrm{kWh}$ and $10 \mathrm{kWh}$, to illustrate QP energy-shifting at a residence. For both battery capacities, let $\chi_{0}=0.5 C$ (initial battery state of charge), and $\bar{B}=-\underline{B}=1 \mathrm{~kW}$ (charge/discharge limits).

Let the load and generation profiles $l$ and $g$ be specified as shown in Fig. 2.3(a), where the residence load includes a utility controlled heated water cylinder [45]. ${ }^{2}$ Both load and generation profiles are specified for $T=24$ hours, $\Delta=30$ minutes and $s=T / \Delta=48$. Additionally, we let the weights $h_{k}=1$ for all $k \in\{1, \ldots, s\}$.

In Fig. 2.3(a) we observe the load profile peaks around midnight, consistent with the utility switching on the allelectric-heated water cylinder at the customer premises, and the generation profile peaks around midday. Consequently the peak generation does not align with the peak load at the residence.

Figure 2.3(b) illustrates the base-line grid profile to which we compare the grid profiles in Fig. 2.3(c)-(d). The baseline grid profile has no battery to time-shift the grid profile $\pi$ appearing in (10) $(C=0 \mathrm{kWh})$. Therefore $\pi$ is calculated directly from the power balance equation in (1) with $\beta_{k}=0$. The grid profiles $\pi$ illustrated in Figure 2.3(c)-(d) arise from the solution of the QP in Lemma 1. Comparing the base-line results in Figure 2.3(b) to the grid profile in Fig. 2.3(c), we observe the $1 \mathrm{~kW}$ battery charges $\left(\beta_{k}<0\right)$ to increase the base-line grid profile (e.g. from $-1.26 \mathrm{~kW}$ to $-0.81 \mathrm{~kW}$ between 11.30 -Midday), and discharges $\left(\beta_{k}>0\right)$ to reduce the base-line grid profile (e.g. from $3.69 \mathrm{~kW}$ to $2.69 \mathrm{~kW}$ between 23.30-Midnight). In Fig. 2.3(d) we observe further reductions in the magnitude of $\pi$, except between 23.30-Midnight, due to the battery discharge constraint of $1 \mathrm{~kW}$.

\footnotetext{
${ }^{2}$ In some countries, residents allow the utility to control their all-electric-heated water systems for periods in the day, given a financial incentive. For these customers, the utility switches their water-heating services on during periods of low load, and off during periods of peak-load, in a manner that ensures minimal impact to the network.
} 

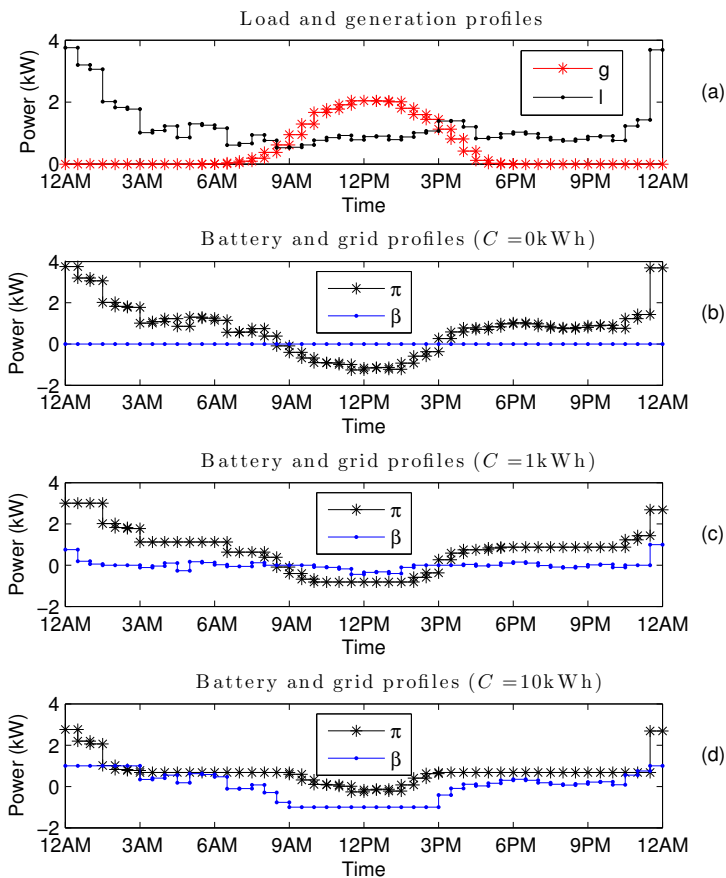

Figure 2: (a) Load and generation profiles $l$ and $g$; (b) grid and battery profiles $\pi$ and $\beta$ for $C=0 \mathrm{kWh}$; (c) grid and battery profiles $\pi$ and $\beta$ for $C=1 \mathrm{kWh}$; (d) grid and battery profiles $\pi$ and $\beta$ for $C=10 \mathrm{kWh}$.

This example demonstrates the reductions in magnitude of the grid profile $\pi$ subject to the battery charge/discharge constraints and capacity $C$. Hence, QP energy-shifting smooths residential load curves when $h_{k}=1$ for all $k \in$ $\{1, \ldots, s\}$. In what follows we design a weighting matrix $\mathbf{H}$ in the QP that reduces residential energy bills, and network peak load corresponding to peak pricing tariffs, while penalizing reverse power flow.

\subsection{Extended definition of grid profile}

We now extend our definition of grid usage over the period $((k-1) \Delta, k \Delta)$ to include explicit reference to the battery capacity $C$ and weights $h_{k}$ as follows:

$$
\pi_{k}^{C}\left(h_{k}\right):=l_{k}-g_{k}-\beta_{k} \quad \text { for all } k \in\{1, \ldots, s\},
$$

where $l_{k}, g_{k}, \beta_{k}$ and $h_{k}$ remain as previously defined. We consequently denote the grid profile over $[0, T]$ by

$$
\pi^{C}(\mathbf{H}):=\left[\pi_{1}^{C}\left(h_{1}\right), \ldots, \pi_{s}^{C}\left(h_{s}\right)\right]^{T} \quad \in \mathbb{R}^{s} .
$$

When battery capacity $C=0$, it follows that

$$
\pi_{k}^{0}=l_{k}-g_{k} \quad \text { for all } k \in\{1, \ldots, s\},
$$

since the battery charging/discharging capacity $\beta_{k}=0, k \in\{1, \ldots, s\}$. The case where $C=0$ is defined as a base-line grid profile against which we compare future grid profiles and is denoted by

$$
\pi^{0}:=\left[\pi_{1}^{0}, \ldots, \pi_{s}^{0}\right]^{T}
$$

We note $\pi^{0}$ is not a function of the selectable weights in $\mathbf{H}$, as the base-line grid profile is solely a function of load and generation profiles in (16). 
Remark 1. The grid profile $\pi$ obtained from solving the quadratic program in Lemma 1 depends not only on the battery constraints $C, \bar{B}, \underline{B}, \chi_{0}$, and selected weightings $h_{k}$, but also the load and generation profiles $l$ and $g$, respectively. Consequently $\pi$ is a function $\pi=\pi\left(l, g, C, \bar{B}, \underline{B}, \chi_{0}, \mathbf{H}\right)$. For notational simplicity, however, we will henceforth omit the functional dependence of $\pi$ on the load/generation profiles and all the battery constraints other than the battery capacity $C$, preferring instead to simply write $\pi^{C}(\mathbf{H})$, where no ambiguity arises. This notational convention reflects our primary degrees of design flexibility, namely battery capacity $C$ and the weighting matrix $\mathbf{H}$.

\section{Billing for a single customer}

In this section we define the energy bill for a single residential customer for the household PV system depicted in Figure 1. To reduce the day-ahead energy bill when the customer uses QP energy-shifting (Lemma 1), we require a financial policy (in $\$ / \mathrm{kWh}$ ) and a battery of capacity $C$. Since the financial policy requires meters in certain locations, with particular modes of operation, we also define the metering topology in Section 3.1.

\subsection{Metering topology}

To formulate the energy bill for a single residential customer, we require the measured power flows from the residential energy system in Figure 1. The metering topology defines how the power flows are to be measured. To formalize the notion of metering topology we define two metering modes in terms of the meters $M \in \mathscr{M}$, and provide an example with respect to meter $M_{2}$ shown in Figure 1.

1. Gross metering mode: We say that meter $M_{2}$ operates in gross metering mode if it measures power flow from node 1 to the battery/load node 2, but not power delivered in the reverse direction. That is, meter $M_{2}$ measures and records only power flows for which $l_{k}-\beta_{k} \geq 0$. In the event $l_{k}-\beta_{k}<0$, the meter records $0 \mathrm{~kW}$. Consequently gross metering mode requires only uni-directional metering.

2. Net metering mode: We say that meter $M_{2}$ operates in net metering mode if it measures power flow in both directions, i.e., from node 1 to the battery/load node $2\left(l_{k}-\beta_{k} \geq 0\right)$, as well as power delivered in the reverse direction (i.e., $\left.l_{k}-\beta_{k}<0\right)$. Consequently net metering mode requires bi-directional metering [46].

The metering topology is defined by the mode of operation (gross or net) of each meter $M \in \mathscr{M}$ in Figure 1 . In order to consider gross metering mode, the direction of power flow must also be included.

The metering topologies considered in this paper are defined below, with the direction of positive power flow as per Figure 1, defined in Section 2.1.

- Metering topology $1: M_{1}$ and $M_{2}$ operate in gross metering mode. $M_{3}$ is not installed. $M_{1}$ measures and records the generation profile $g_{k} \geq 0$ for all $k, M_{2}$ measures and records the power flow $l_{k}-\beta_{k} \geq 0$ for all $k$.

- Metering topology 2: $M_{3}$ operates in net metering mode. $M_{1}$ and $M_{2}$ are not installed.

\subsection{Financial policies}

To calculate the energy bill for a single residential customer, we require the measured power flows from the residential energy system in Figure 1, and the corresponding electricity prices. Our definition of a financial policy (in $\$ / \mathrm{kWh}$ ) formalizes the electricity prices and includes incentives intended to influence customer energy utilization. Example incentives include time-of-use (TOU) pricing, feed-in-tariffs and net metering [3], [46]. Our definition of a financial policy below is sufficiently general to include these incentives in addition to more sophisticated dynamic day-ahead pricing rates $[23,29,37]$

Our definition of a financial policy requires an electricity billing profile and an electricity compensation profile over $[0, T]$, for each installed meter in $\mathscr{M}$. The direction of power flow associated with electricity billing/compensation is defined with reference to the direction of positive power flow that is specified at each meter $M \in \mathscr{M}$. We denote electricity billing (in $\$ / \mathrm{kWh}$ ) at meter $M \in \mathscr{M}$ over the period $((k-1) \Delta, k \Delta)$ by $\eta_{k}^{b}(M)$ for all $k \in\{1, \ldots, s\}$, and 
define the electricity billing profile over $[0, T]$ at $M$ as $\eta^{b}(M):=\left[\eta_{1}^{b}(M), \ldots, \eta_{s}^{b}(M)\right]^{T} \in \mathbb{R}_{>0}^{s}$. Likewise we denote the electricity compensation (in $\$ / \mathrm{kWh}$ ) at meter $M \in \mathscr{M}$ over the period $((k-1) \Delta, k \Delta)$ by $\eta_{k}^{c}(M)$ for all $k \in\{1, \ldots, s\}$, and define the electricity compensation profile over $[0, T]$ at $M$ as $\eta^{c}(M):=\left[\eta_{1}^{c}(M), \ldots, \eta_{s}^{c}(M)\right]^{T} \in \mathbb{R}_{\geq 0}^{s}$.

In order to implement a financial policy, certain types of meters are required in particular locations. For example a financial policy may require the meter $M_{1}$ (in Fig. 1), which records positive power flows from the solar PV to node 1. For this meter the financial policy will specify the electricity billing and compensation profiles $\eta^{b}\left(M_{1}\right), \eta^{c}\left(M_{1}\right)$, respectively. If the electricity billing (or compensation) profile at meter $M_{1}$ is defined by $\eta_{k}^{b}\left(M_{1}\right)=0$ (or $\left.\eta_{k}^{c}\left(M_{1}\right)=0\right)$ for all $k \in\{1, \ldots, s\}$, then it is sufficient that meter $M_{1}$ operates in gross metering mode. In this case the power flow to be measured is in the same direction specified for electricity compensation (or billing).

We now define a financial policy over $[0, T]$ by using the day ahead electricity billing and compensation profiles at each installed meter in $\mathscr{M}$. An example financial policy is defined with reference to Fig. 1 for $\mathscr{M}=\left\{M_{1}, M_{2}, M_{3}\right\}$. The direction of positive power flow at meter $M_{1}$ is defined by $g$ (from the solar PV to node 1) and electricity is compensated in this direction $\eta^{c}\left(M_{1}\right)$. The direction of positive power flow at meter $M_{2}$ is defined by $l-\beta \geq 0$ (from node 1 to node 2) and electricity is billed in this direction $\eta^{b}\left(M_{2}\right)$. The direction of positive power flow at meter $M_{3}$ is defined by $\pi$ (from the PCC to node 1) and electricity is billed in this direction $\eta^{b}\left(M_{3}\right)$. For each electricity compensation (or billing) profile $\eta^{b}(M)$ (or $\eta^{c}(M)$ ), there also exists an electricity billing (or compensation) profile $\eta^{c}(M)$ (or $\eta^{b}(M)$ ) for power flowing against the positive direction at meter $M \in \mathscr{M}$.

The financial policies considered in this paper are defined with reference to metering topologies 1 and 2 defined in Section 3.1. The financial policy associated with metering topology 1 includes an electricity compensation profile at meter $M_{1}$ (for power flow from the solar PV to node 1), and an electricity billing profile at meter $M_{1}$ (for power flows in the reverse direction), represented by $\eta^{c}\left(M_{1}\right)$ and $\eta^{b}\left(M_{1}\right)$ respectively; and an electricity compensation profile at meter $M_{2}$ (for power flow from node 2 to node 1), and an electricity billing profile at meter $M_{2}$ (for power flows from node 1 to node 2), represented by $\eta^{c}\left(M_{2}\right)$ and $\eta^{b}\left(M_{2}\right)$ respectively. Furthermore, $\eta_{k}^{b}\left(M_{1}\right)=0$ and $\eta_{k}^{c}\left(M_{2}\right)=0$, for all $k \in\{1, \ldots, s\}$ and hence it is sufficient that meters $M_{1}$ and $M_{2}$ operate in gross metering mode, as per the definition of metering topology 1 .

The financial policy associated with metering topology 2 has an electricity compensation profile at meter $M_{3}$ (for power flow from node 1 to PCC) and an electricity billing profile at meter $M_{3}$ (for power flow from the PCC to node 1), represented by $\eta^{c}\left(M_{3}\right)$ and $\eta^{b}\left(M_{3}\right)$ respectively. The following table summarizes the electricity billing and compensation profiles for metering topologies 1 and 2 .

Table 1: Electricity billing and compensation profiles for metering topologies 1 and 2

\begin{tabular}{|c|c|c|c|c|}
\hline Meter & \multicolumn{2}{|c|}{ Metering Topology 1 } & \multicolumn{2}{c|}{ Metering Topology 2 } \\
\hline & billing & compensation & billing & compensation \\
\hline$M_{1}$ & $\eta^{b}\left(M_{1}\right)=\mathbf{0}$ & $\eta^{c}\left(M_{1}\right)$ & & \\
\hline$M_{2}$ & $\eta^{b}\left(M_{2}\right)$ & $\eta^{c}\left(M_{2}\right)=\mathbf{0}$ & & \\
\hline$M_{3}$ & & & $\eta^{b}\left(M_{3}\right)$ & $\eta^{c}\left(M_{3}\right)$ \\
\hline
\end{tabular}

To implement a gross feed-in tariff, we observe metering topology 1 is sufficient. To implement a net feed-in tariff, or net metering, we observe metering topology 2 is sufficient.

\subsection{Energy bill}

To define the energy bill for the residential energy system in Figure 1, we combine the financial policy (in $\$ / \mathrm{kWh}$ ) with the measured power flows defined in Section 2.1. To reduce the energy bill when QP energy-shifting, we seek a weighting matrix $\mathbf{H}$ given a fixed battery capacity $C$.

In what follows we define the energy bill (in \$/day) in terms of the respective financial policy associated with metering topologies 1 and 2 (Section 3.2). We assume the day-ahead billing and compensation profiles in the respective financial policies are fixed by the utility or regulatory body and available to the consumer. 
In equation (6) we constrained the initial and final states of charge of the battery to be equal. Consequently, we assume the cost associated with charging the battery to $\chi_{0}$ can be compensated for with the remaining charge at the end of the day $\chi_{s}$. Therefore, in defining of the energy bill, we ignore the cost associated with charging the battery to an initial state of charge.

To formalize the energy bill associated with metering topology 1 , we select the electricity prices that correspond to measured power flows at meters $M_{1}$ and $M_{2}$. That is, for the financial policy relating to metering topology 1 , we define $\sigma_{k}\left(M_{1}\right)$ and $\sigma_{k}\left(M_{2}\right)$ as follows:

$$
\begin{gathered}
\sigma_{k}\left(M_{1}\right)= \begin{cases}\eta_{k}^{c}\left(M_{1}\right), & \text { if } g_{k} \geq 0 \\
\eta_{k}^{b}\left(M_{1}\right), & \text { if } g_{k}<0,\end{cases} \\
\sigma_{k}\left(M_{2}\right)= \begin{cases}\eta_{k}^{b}\left(M_{2}\right), & \text { if } l_{k}-\beta_{k} \geq 0 \\
\eta_{k}^{c}\left(M_{2}\right), & \text { if } l_{k}-\beta_{k}<0,\end{cases}
\end{gathered}
$$

and denote $\sigma\left(M_{1}\right):=\left[\sigma_{1}\left(M_{1}\right), \ldots, \sigma_{s}\left(M_{1}\right)\right]^{T} \in \mathbb{R}_{\geq 0}^{s}$ and $\sigma\left(M_{2}\right):=\left[\sigma_{1}\left(M_{2}\right), \ldots, \sigma_{s}\left(M_{2}\right)\right]^{T} \in \mathbb{R}_{\geq 0}^{s}$ over the period $[0, T]$. Recall $\eta_{k}^{b}\left(M_{1}\right)=0$ and $\eta_{k}^{c}\left(M_{2}\right)=0$, for all $k \in\{1, \ldots, s\}$.

In order to minimize the energy bill associated with metering topology 1, we choose the weighting matrix for a given battery capacity with constraints (8)-(9) known and fixed as

$$
\mathbf{H}_{1}:=\mathbf{H}\left(\sigma\left(M_{1}\right), \sigma\left(M_{2}\right)\right) .
$$

Hence the choice of weighting matrix in the cost function (11) is dependent on the implemented financial policy.

Having fixed $\mathbf{H}_{1}$ in equation (20), we define the residential energy bill associated with metering topology 1, denoted by $\Sigma^{C}\left(\mathbf{H}_{1}\right)$ (in $\$ /$ day) by

$$
\Sigma^{C}\left(\mathbf{H}_{1}\right):=\Delta\left((l-\beta)^{T} \sigma\left(M_{2}\right)-g^{T} \sigma\left(M_{1}\right)\right) .
$$

When the battery capacity $C=0$, the energy bill defined in (21) reduces to

$$
\Sigma^{0}:=\Delta\left(l^{T} \eta^{b}\left(M_{2}\right)-g^{T} \eta^{c}\left(M_{1}\right)\right)
$$

since the battery charging/discharging capacity $\beta_{k}=0$ for all $k \in\{1, \ldots, s\}$, rendering the selectable weights in $\mathbf{H}_{1}$ irrelevant. The case where $C=0$ also serves as a base-line energy bill, which we use as a comparison when assessing the financial benefits of battery storage.

Remark 2. The energy bill notation convention $\Sigma^{C}\left(\mathbf{H}_{1}\right)$ is simplified, and consistent with the suppression of functional dependence described in Remark 1. That is, our notation reflects our primary degrees of design flexibility, the battery capacity $C$ and the weighting matrix.

To formalize the energy bill associated with metering topology 2, we select the electricity prices that correspond to measured power flows at meter $M_{3}$. That is, we define $\sigma_{k}\left(M_{3}\right)$ in terms of the financial policy as

$$
\sigma_{k}\left(M_{3}\right)= \begin{cases}\eta_{k}^{b}\left(M_{3}\right), & \text { if } \pi_{k}^{C}\left(h_{k}\right) \geq 0 \\ \eta_{k}^{c}\left(M_{3}\right), & \text { if } \pi_{k}^{C}\left(h_{k}\right)<0\end{cases}
$$

and we denote $\sigma\left(M_{3}\right):=\left[\sigma_{1}\left(M_{3}\right), \ldots, \sigma_{s}\left(M_{3}\right)\right]^{T} \in \mathbb{R}_{\geq 0}^{s}$ over the period $[0, T]$. In order to minimize the energy bill associated with metering topology 2 , we choose the weighting matrix for a given battery capacity with constraints (2)-(4) known and fixed as

$$
\mathbf{H}_{2}:=\mathbf{H}\left(\sigma\left(M_{3}\right)\right)
$$

Having fixed $\mathbf{H}_{2}$ in (24), we define the energy bill associated with the financial policy relating to metering topology 2 by

$$
\Sigma^{C}\left(\mathbf{H}_{2}\right):=\Delta \pi^{C}\left(\mathbf{H}_{2}\right)^{T} \sigma\left(M_{3}\right)
$$


which reduces to the base-line energy bill for $C=0$ given by

$$
\Sigma^{0}:=\Delta(l-g)^{T} \sigma\left(M_{3}\right)
$$

where $\pi^{0}=l-g$ since the battery charging/discharging capacity satisfies $\beta_{k}=0$ for all $k \in\{1, \ldots, s\}$.

\section{Savings for a single customer}

In this section we define the energy savings for the household PV system depicted in Figure 1. The results in this section allow a single customer to assess the cost-effectiveness of installing a battery of a given size. Recall, this paper focuses on the operational energy savings associated with QP energy-shifting and as such we omit the capital cost of installing a battery.

\subsection{Energy savings}

To examine the effectiveness of QP energy-shifting for a given size battery, we define the energy savings (in \$/day). The energy savings are denoted by $\Psi^{C}(\mathbf{H})$ and defined by

$$
\Psi^{C}(\mathbf{H}):=\Sigma^{0}-\Sigma^{C}(\mathbf{H}) .
$$

We recall from Section 3.3, the energy bill $\Sigma^{C}(\mathbf{H})$ is defined for a particular financial policy and selection of weights in $\mathbf{H}$, given load and generation profiles $l$ and $g$, a battery of a given size $C$, with constraints (2)-(4) known and specified. When $C=0, \Sigma^{0}$ denotes the base-line energy bill.

Given unique load and generation profiles for 365 consecutive days and a battery of a given size $C$, with constraints (2)-(4) known and specified, their exists a unique energy saving $\Psi^{C}(\mathbf{H})$ for each of the 365 days. We define the summation of these unique energy savings by $\Theta^{C}(\mathbf{H})$ in $\$ / y \mathrm{r}$ and label this summation the annual savings. Thus when the annual savings are positive, there exists an operational benefit to QP energy-shifting.

Hence $\Theta^{C}(\mathbf{H})>0$ implies the installation is operationally cost-effective, $\Theta^{C}(\mathbf{H})=0$ implies the installation is operationally cost-neutral and $\Theta^{C}(\mathbf{H})<0$ implies no financial benefit for battery storage for that given year.

\subsection{Special case: zero energy savings}

Consider the special case where there is a fixed price for electricity (in $\$ / \mathrm{kWh}$ ) at all installed meters in $\mathscr{M}$, irrespective of power flow direction and time of day. Lemma 2 below demonstrates that under these circumstances, there is no financial incentive for a resident to install battery storage. That is, since the battery acts as an energy time-shifter, the lack of differential pricing at any point in time gives no incentive to energy time-shift.

Lemma 2. Fix $\eta>0$ and let the electricity billing and compensation profiles in the financial policy satisfy the following for all $M \in \mathscr{M}$ :

$$
\begin{aligned}
& \eta_{j}^{b}=\eta_{k}^{c}=\eta \quad \text { for all } j, k \in\{1, \ldots, s\}, \\
& \eta^{b}=\eta^{c}=\eta \mathbb{1} .
\end{aligned}
$$

Assume all meters $M \in \mathscr{M}$ are installed such that all power flowing to or from the grid is quantifiable (for example metering topology 1 or 2). Then for all choices of battery capacity $C$ and weighting matrix $\mathbf{H}$, the energy savings are $\Psi^{C}(\mathbf{H})=0$.

Proof. Consider metering topology 2. Rearranging equation (3) yields

$$
\chi_{0}-\chi_{s}=\sum_{k=1}^{s} \beta_{k} \Delta .
$$


Recall from the definition of the time window $T$, we require $\Delta$ to be positive $(\Delta>0)$ and a constant. Combining the definition of $\Delta$ with the constraint in equation (6) implies

$$
\sum_{k=1}^{s} \beta_{k}=\beta^{T} \mathbb{1}=0
$$

Furthermore, definitions (14) and (15) imply

$$
\pi^{C}\left(\mathbf{H}_{2}\right)=l-g-\beta
$$

Additionally, substituting equations (28)-(29) into (23) yields

$$
\sigma\left(M_{3}\right)=\eta \mathbb{1}
$$

Therefore, from equation (25) the energy bill is

$$
\Sigma^{C}\left(\mathbf{H}_{2}\right)=\Delta \pi^{C}\left(\mathbf{H}_{2}\right)^{T} \sigma\left(M_{3}\right)=\Delta(l-g-\beta)^{T} \eta \mathbb{1}=\Delta(l-g)^{T} \eta \mathbb{1}-\Delta \eta \beta^{T} \mathbb{1}=\Delta(l-g)^{T} \sigma\left(M_{3}\right)=\Sigma^{0},
$$

where the final equality is defined in equation (26).

The energy savings (27) are then

$$
\Psi^{C}\left(\mathbf{H}_{2}\right)=\Sigma^{0}-\Sigma^{C}\left(\mathbf{H}_{2}\right)=0 .
$$

A similar calculation can be performed for other metering topologies, provided the meters in $\mathscr{M}$ are installed such that all power flowing to or from the grid is quantifiable.

\section{Heuristic for selecting the weighting matrix}

In this paper our objective is to maximize the daily operational savings that accrue to a single customers, while penalizing large voltage swings observed in the distribution network stemming from reverse power flow and peak load. We assume peak electricity billing rates coincide with generation shortages or peak grid demand and look to prioritize the minimization of energy flow from the grid during these events, while penalizing reverse power flow. To achieve our objective, we seek a weighting matrix $\mathbf{H}$ in the QP for a single customer with battery storage in the residential setting shown in Fig. 1. Given perfect day-ahead load and generation forecasts, and battery constraints (8)-(9), the HEM system computes the weighting matrix $\mathbf{H}$ via a heuristic for each day-ahead. We define the heuristic in what follows.

In Section 2.2, the minimization of expression (10) was presented as a constrained quadratic program (Lemma 1), where the weights $h_{k}$ in $\mathbf{H}$ were selectable. In this section we consider the specification of the matrix $\mathbf{H}$ that maximizes the annual savings, while reducing the impact of the residential system on the grid. In practice, the matrix $\mathbf{H}$ is difficult to obtain, as it depends on a variety of factors including financial policies, metering topologies and daily variations in load and generation profiles. To address this problem we propose a greedy-search heuristic for obtaining a so-called preferred $\mathbf{H}$, which is in turn based upon a base-line weighting matrix denoted by $\mathbf{H}_{0}$.

When selecting the weights in the preferred $\mathbf{H}$, our rationale is to increase base-line weights when electricity billing is high and decrease base-line weights when electricity billing is low, and to continue increasing/decreasing so long as the daily residential savings increase. This rational reduces network peak loads without contributing to reverse power flow during the peak pricing period, and increases operational savings that accrue to customers.

The basic idea of the heuristic is to increase each weight $h_{k}$ in $\mathbf{H}_{0}$, as long as this increase leads to an increased energy saving in (27). To mitigate against numerical difficulties with the solution of the quadratic program in Lemma 1 , we increase weights in $\mathbf{H}_{0}$ until a maximum allowable value of $h_{k}$ is reached. To this end, weights in $\mathbf{H}_{0}$ are scaled 
by the minimum cost and capped at a maximum value. To cap the weights $h_{k}$ we introduce the following saturation operation:

$$
\operatorname{sat}_{1}^{\bar{h}}\left(h_{k}\right):= \begin{cases}1, & \text { if } h_{k}<1 \\ h_{k}, & \text { if } 1 \leq h_{k} \leq \bar{h} \\ \bar{h}, & \text { if } h_{k}>\bar{h},\end{cases}
$$

where the lower bound is 1 in accordance with the definition of $h_{k}$ in Section 2.2 and $\bar{h}>1$ is fixed. The constant $\bar{h}$ is chosen to mitigate against numerical difficulties in solving the QP in Lemma 1 . In this paper, we set $\bar{h}=1000$.

To formalize the definition of the base-line weighting matrix, let

$$
\begin{aligned}
\tilde{\eta}_{k} & :=\sum_{M \in \mathscr{M}} \eta_{k}^{b}(M), & \forall k \in\{1, \ldots, s\} \\
\eta^{\star}: & =\min _{k \in\{1, \ldots, s\}} \tilde{\eta}_{k}, &
\end{aligned}
$$

and define the base-line weighting matrix $\mathbf{H}_{0}$ as

$$
\mathbf{H}_{0}:=\operatorname{diag}\left[\mathbf{H}_{0}^{(1)}, \ldots, \mathbf{H}_{0}^{(k)}, \ldots, \mathbf{H}_{0}^{(s)}\right]
$$

where $\mathbf{H}_{0}^{(k)}:=\operatorname{sat}_{1}^{\bar{h}}\left(\tilde{\eta}_{k} / \eta^{\star}\right)$.

Given $\mathbf{H}_{0}$, the proposed heuristic requires the function for energy savings $\Psi(\cdot)$ defined in (27). Recall the energy savings function $\Psi(\cdot)$ requires the constraints and solution to the QP in Lemma 1 and the energy bill $\Sigma(\cdot)$ pertaining to a given metering topology and financial policy as defined in Section 3. To simplify the notation, we use $\Psi(\cdot)$ rather than $\Psi^{C}(\mathbf{H})$ to indicate that the battery capacity $C$ is fixed.

The main loop in the heuristic below (lines 6-19), doubles weights in $\mathbf{H}_{0}$ progressively, from the largest to the smallest element in $\mathbf{H}_{0}$. If there exist multiple elements in $\mathbf{H}_{0}$ with the same magnitude, we double the multiple elements concurrently. The set of live indices $\tilde{s}$ keeps track of the indices in $\mathbf{H}_{0}$ that are yet to be increased, and $\mathbf{I}^{\tilde{s}}$ denotes an $s$-by-s matrix in which $\mathbf{I}_{j, j}^{\tilde{s}}=1$ if $j \in \tilde{s}$ and zero otherwise.

\section{Application of QP energy-shifting}

We analyzed measured load and generation profiles from July 1st 2010 to the 30th of June 2011, for each of 300 randomly selected low voltage customers located in an Australian distribution network, operated by Ausgrid. The Ausgrid distribution network covers $22,275 \mathrm{~km}^{2}$ and includes load centers in Sydney and regional New South Wales. The load and generation profiles $l$ and $g$ for each of the 300 customers are defined with $T=24$ hours, $\Delta=30$ minutes, and $s=T / \Delta=48$, for each day in the 365 days.

We eliminated customers with a maximum load or PV generation less than $5 \mathrm{~W}$ on any day of the year $\left(l_{k}<0.005\right.$ or $g_{k}<0.005$ for all $k \in\{1, \ldots, s\}$ ), leaving 145 of the original 300 customers. We refer to this set of 145 customers as the ensemble.

When QP energy-shifting, the annual savings for each customer in the ensemble are dependent on a variety of factors and we investigate four of the most important factors in what follows. In Section 6.2 we investigate the influence of daily variations in the load and generation profiles on savings for particular customers in the ensemble. In Section 6.3 we compare annual savings for different metering topologies. In Section 6.4 we compare annual savings with and without the preferred H. In Section 6.5 we investigate the influence of battery capacity on annual savings. The computational time when QP energy-shifting (including the time to find the preferred $\mathbf{H}$ ) on each day for each ensemble member is on average 0.422 seconds with an Intel i7-2630QM processor. 


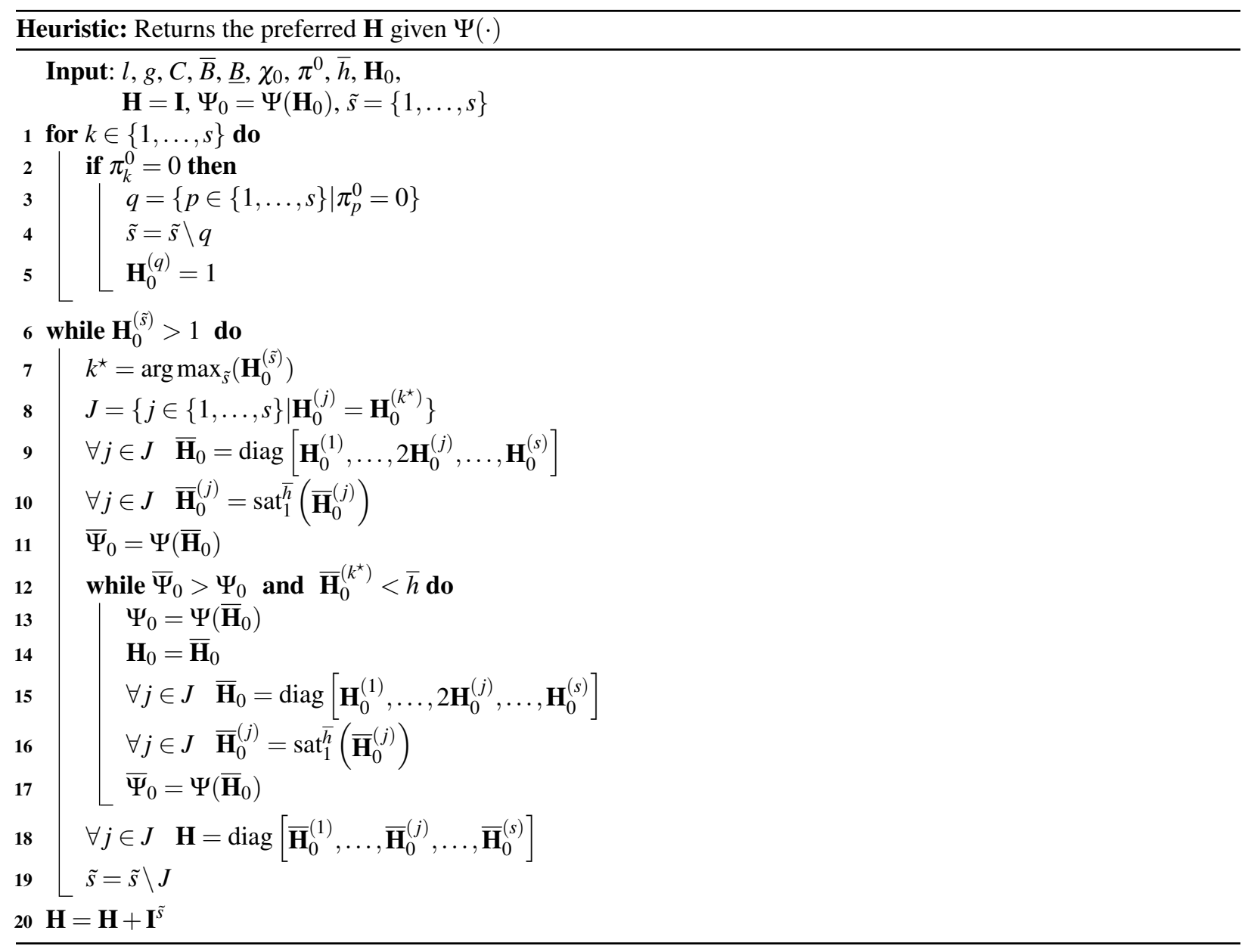




\subsection{Simulation Parameters}

In the following we use the heuristic to find the $\mathbf{H}$ matrix when QP energy-shifting, except as specified in Section 6.4. To calculate the annual savings for the ensemble when QP energy-shifting, we fix the battery capacity at $10 \mathrm{kWh}$, except in Section 6.5 where we vary the battery capacity within the range $0 \mathrm{kWh} \leq C \leq 30 \mathrm{kWh}$. In all cases, the remaining battery constraints (2)-(4) are chosen as $\chi_{0}=0.5 C$, and $\bar{B}=-\underline{B}=5 \mathrm{~kW}$. We also fix the length-s billing and compensation profiles (each given in $\$ / \mathrm{kWh}$ ) for metering topology 1 as follows:

$$
\begin{aligned}
\eta^{b}\left(M_{1}\right) & =\eta^{c}\left(M_{2}\right)=[0, \ldots, 0]^{T} \\
\eta^{c}\left(M_{1}\right) & =[0.4, \ldots, 0.4]^{T} \\
\eta^{b}\left(M_{2}\right) & =\left[\ldots, \eta_{k}^{b}, \ldots\right]^{T}
\end{aligned}
$$

where $\eta_{1-14}^{b}=0.03, \quad \eta_{15-28}^{b}=0.06, \quad \eta_{29-40}^{b}=0.3, \quad \eta_{41-44}^{b}=0.06$, and $\eta_{45-48}^{b}=0.03$. The non-zero profiles are shown in Figure 3(a).

For metering topology 2 , the length-s compensation and billing profiles (in $\$ / \mathrm{kWh}$ ) are again fixed and given by

$$
\begin{aligned}
& \eta^{c}\left(M_{3}\right)=[0.4, \ldots, 0.4]^{T}, \\
& \eta^{b}\left(M_{3}\right)=\left[\ldots, \eta_{k}^{b}, \ldots\right]^{T},
\end{aligned}
$$

such that $\eta^{b}\left(M_{3}\right)=\eta^{b}\left(M_{2}\right)$. The non-zero profiles are shown in Figure 3(b).
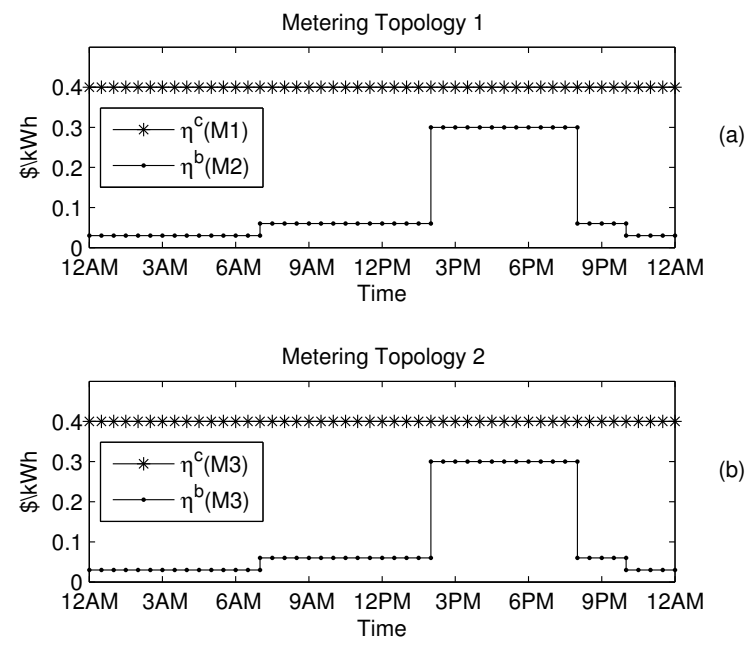

Figure 3: Non-zero billing and compensation profiles for metering topologies 1 and 2.

For both metering topologies in Figure 3 we describe electricity billing from 10pm-7am at the rate of $\$ 0.03 / \mathrm{kWh}$ as an off-peak pricing period, electricity billing from $7 \mathrm{am}-2 \mathrm{pm}$ and again from $8 \mathrm{pm}-10 \mathrm{pm}$ at the rate of $\$ 0.06 / \mathrm{kWh}$ as a shoulder pricing period and electricity billing from $2 \mathrm{pm}-8 \mathrm{pm}$ at the rate of $\$ 0.30 / \mathrm{kWh}$ as a peak pricing period.

For metering topology 1 in Figure 3 we describe electricity compensation $\eta^{c}\left(M_{1}\right)$ as a gross feed-in tariff. For metering topology 2 in Figure 3 we describe electricity compensation $\eta^{c}\left(M_{3}\right)$ as a net feed-in tariff.

\subsection{Influence of Load and Generation Profiles}

In this section we identify typical load and generation profiles that result in either a positive or negative operational saving when QP energy-shifting under metering topology 1. To do this we compare daily energy savings for two 
customers in the ensemble. The selected two customers are chosen with significant differences in their respective load and generation profiles $(l$ and $g$ ). The two representative customers are denoted Customer 75 and Customer 200.

Figure 4 illustrates the significant differences in the respective load and generation profiles for customers 75 and 200. In Figure 4(a) we observe Customer 75 consumed most of its energy during the off-peak pricing period between $10 \mathrm{pm}$ and $7 \mathrm{am}$. Meanwhile the solar PV unit delivered energy from 7am to 7:30pm and was in excess of the residential energy demand from 8am-7:30pm. Consequently, Customer 75 delivered energy to the grid from 8am-7:30pm on the 9th of January 2011.

In Figure 4(b) we observe Customer 200 consumed a significant proportion of its energy during the peak pricing period $(2 \mathrm{pm}-8 \mathrm{pm})$ and very little energy during the off-peak pricing period (10pm - 7am). In Figure 4(b) we also observe the generation profile is less than the load profile for the entire day $\left(g_{k}<l_{k}\right.$ for all $\left.k \in\{1, \ldots, s\}\right)$. Consequently, there was no energy delivered to the grid by Customer 200 on the 5th of July 2010.
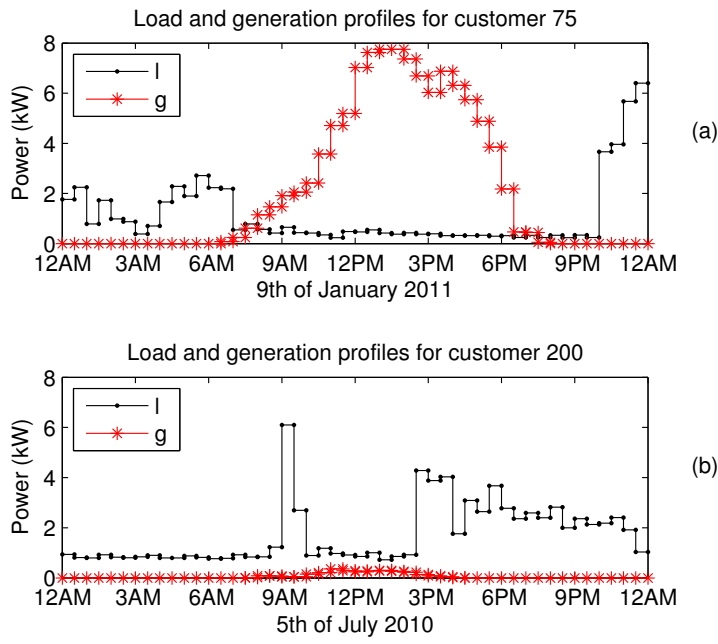

Figure 4: Representative load and generation profiles for customers 75 and 200.

For both customers 75 and 200, given a financial policy associated with metering topology 1 and simulation parameters defined in Section 6.1, we calculate the daily energy savings given a $10 \mathrm{kWh}$ battery. From these daily energy savings we find Customer 75 would have lost $\$ 2.68$ on the 9th of January 2011 and Customer 200 would have saved $\$ 2.70$ on the 5th of July 2010 by using QP energy-shifting.

To understand why QP energy-shifting would save Customer $200 \$ 2.70$, while costing Customer $75 \$ 2.68$, given the load and generation profiles in Figure 4, we compare the respective battery states of charge. In Figure 5(a) we observe the battery discharges mostly during the off-peak pricing period when Customer 75 consumed most of its energy and charges during the peak pricing period rather than the shoulder pricing period when PV generation was high and load low due to the weightings imposed via the heuristic. Consequently the cost of charging the battery is not offset by the cost of discharging the battery for Customer 75 on the 9th of January 2011.

In Figure 5(b) we observe the battery discharges mostly during the peak pricing period when the customer consumed most of its energy and charges during the off-peak pricing period as well as when the solar PV generated energy. Therefore the cost of charging the battery is offset by the cost of discharging the battery for Customer 200 on the 5 th of July 2010. 

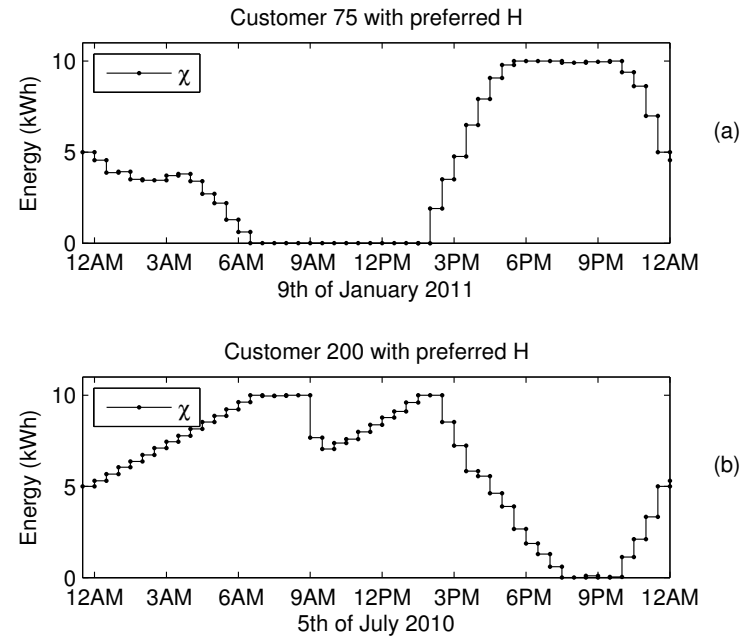

Figure 5: The battery SOC for customers 75 and 200 when using QP energy-shifting on the 9th of January 2011 and the 5th of July 2010, respectively.

On each day from the 1 st of July 2010 to the 30th of June 2011 we calculated the daily energy savings for customers 75 and 200. Figure 6 illustrates the distribution of these daily savings. In Figure 6(a) we observe QP energy-shifting results in Customer 75 losing money over the course of a year, even though some days provide savings. This loss of money is attributed to load and generation profiles that caused the battery to charge during peak pricing periods and discharge during off-peak pricing periods, consistent with our observations in Figure 5(a).

In Figure 6(b) we observe QP energy-shifting results in Customer 200 saving money over the course of a year. This saving is attributed to load and generation profiles that cause the battery to charge during the off-peak pricing periods and discharge during the peak pricing periods, consistent with our observations in Figure 5(b).

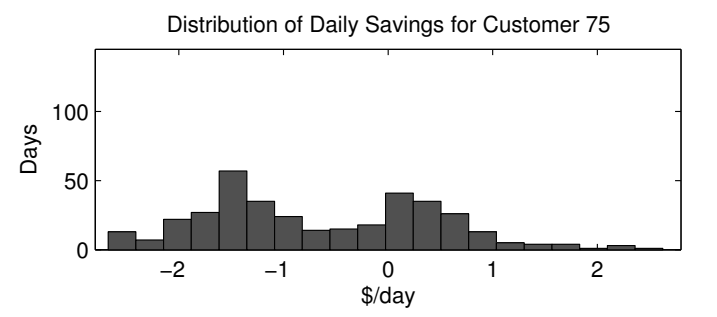

(a)

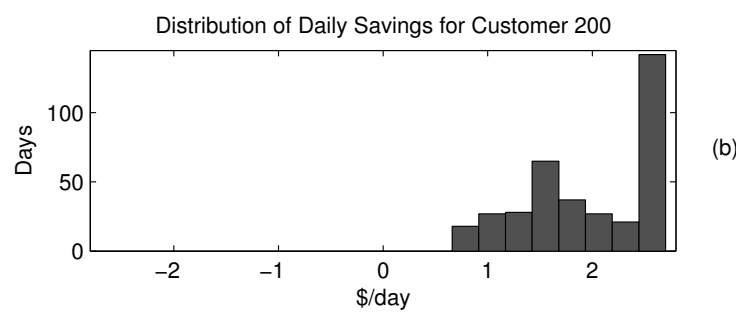

Figure 6: Distribution of daily saving resulting form QP energy-shifting for customers 75 and 200

Consequently, given metering topology 1 , the daily cost of charging a given battery must be offset by the daily cost of discharging a given battery for a customer to reap the benefits of QP energy-shifting. Therefore, given the financial 
policy associated with metering topology 1 described in Section 6.1, customers that consume most of their energy during the off-peak pricing period and generate more energy then they consume, will not financially benefit from QP energy-shifting. On the other hand, those who consume most of their energy during the peak pricing period and generate less energy then they consume, will financially benefit from QP-energy shifting.

\subsection{Influence of metering topologies}

For each customer in the ensemble, we now calculate and compare the annual savings associated with QP energyshifting given the financial policy associated with either metering topology 1 or 2 as presented in Section 3.1. For each customer, the annual savings are again calculated for a $10 \mathrm{kWh}$ battery and in all cases, the simulation parameters are as defined in Section 6.1.

Figure 7 illustrates the distribution of annual savings for all customers in the ensemble under two metering topologies. In Figure 7(a) we observe metering topology 1 saves the ensemble on average $\$ 350 / y$, however nine customers lose money, including Customer 75. In Figure 7(b) we observe metering topology 2 saves the ensemble on average $\$ 100 / y r$, however fifty customers lose money, including Customer 75 . Hence some customers do not benefit from QP energy-shifting, irrespective of the metering topology.
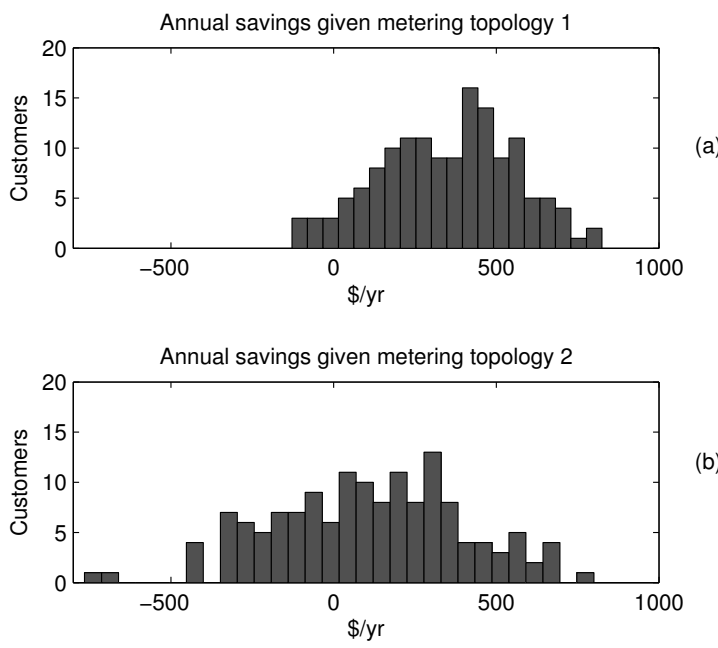

Figure 7: Annual savings distribution for the ensemble resulting from metering topologies 1 and 2

We again visit the representative load and generation profiles for Customer 75 in Figure 4(a) to understand the underlying principles that result in Customer 75 not benefiting from QP energy-shifting, given metering topology 2. In Figure 4(a) we observed Customer 75 generated energy and delivered most of this energy to the grid and was compensated for this at $\$ 0.4 / \mathrm{kWh}$. However if Customer 75 employed QP energy-shifting, the generated energy would instead charge a battery when in excess of the load, leading to a loss in compensation at $\$ 0.4 / \mathrm{kWh}$. Given the financial policy relating to metering topology 2 , it is not possible for this customer to recoup this compensation loss by discharging the battery, as the maximum billing rate is $\$ 0.3 / \mathrm{kWh}$. Therefore, customers that would ordinarily deliver energy to the grid, may not profit from QP energy-shifting when electricity compensation is in excess of electricity billing.

\subsection{Influence of the selection of $\mathbf{H}$}

In this section we verify the heuristic proposed in Section 5. The heuristic finds a matrix $\mathbf{H}$ that increases the annual savings, with comparison to the base-line $\mathbf{H}$ (denoted $\mathbf{H}_{0}$ ). The results in this section are based on the battery constraints and financial policies as per section 6.1. 
For each customer in the ensemble we calculate the annual savings when QP energy-shifting using both the preferred $\mathbf{H}$ and $\mathbf{H}_{0}$ given the financial policies associated with metering topologies 1 and 2 . We then average the annual savings of the ensemble and label this average the mean annual savings (in \$/yr).

We record the mean annual savings for both the preferred $\mathbf{H}$ and $\mathbf{H}_{0}$ in Table 2. From this table we observe the preferred $\mathbf{H}$ increases mean annual savings when QP energy-shifting, irrespective of the metering topology. Hence the heuristic given in Section 5 successfully increases the mean annual savings for the ensemble, with comparison to $\mathbf{H}_{0}$.

Table 2: Mean Annual Savings for the ensemble

\begin{tabular}{|c|c|c|c|c|}
\hline Battery & \multicolumn{2}{|c|}{ Metering Topology 1} & \multicolumn{2}{c|}{ Metering Topology 2} \\
\hline & $\mathbf{H}_{0}$ & $\mathbf{H}$ & $\mathbf{H}_{0}$ & $\mathbf{H}$ \\
\hline $\mathbf{1 0 k W h}$ & $\$ 266 / \mathrm{yr}$ & $\$ 348 / \mathrm{yr}$ & $\$ 9 / \mathrm{yr}$ & $\$ 90 / \mathrm{yr}$ \\
\hline
\end{tabular}

\subsection{Influence of the battery capacity}

To assess the influence of battery capacity (in $\mathrm{kWh}$ ) on residential annual savings when QP energy-shifting we consider again customers 75 and 200 from the ensemble. For these customers we vary the battery capacity $(C)$ given the set of battery capacities (in kWh) $C=\{0,0.1,1,2,4,6,8,10,15,20,30\}$ and plot the results in Figure 8.

In Figure 8 we observe that an increase in the battery capacity results in an increase in financial losses for Customer 75. In comparison, an increase in battery capacity results in an increase in annual savings for Customer 200. Furthermore, the increase in annual savings for Customer 200 rapidly approaches an asymptotic value with a 30kWh battery providing minimal additional savings over a $15 \mathrm{kWh}$ battery. Consequently, not all customers benefit from battery storage and increasing the size of the battery does not necessarily increase the annual savings for a given customer.

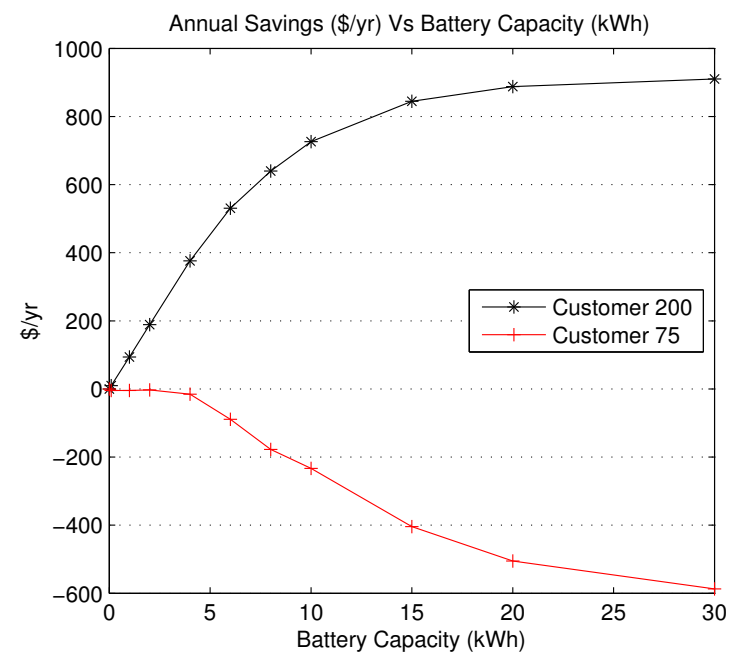

Figure 8: Comparison of annual savings for customers 200 and 75 with variable battery capacities, under the financial policy pertaining to metering topology 1 .

Considering the subset of customers who do financially benefit from QP energy-shifting, if we know the capital cost of installing a battery of a given size, with constraints known and specified, Figure 8 may be useful in identifying the most cost-effective battery capacity. Furthermore, given the capital cost of installing a battery, we expect there also exists a critical annual saving where increases in battery capacity may no longer be cost-effective. 


\section{Conclusions}

In this paper we have presented a QP-based algorithm for day-ahead scheduling of residential battery storage colocated with solar PV. The QP-based algorithm is formulated to balance two objectives. The first objective is to minimize the impact of the residential system on the grid, by reducing the network peak demand and non-compliant voltage deviations associated with reverse power flow. The second objective is to increase the daily operational savings that accrue to customers, by time-shifting residential load from peak pricing periods to off-peak pricing periods. In particular, we balance the reduction of load during during peak pricing periods with penalties for reverse power flow during the same period so that voltage rise associated with solar PV is not simply time-shifted to the peak pricing periods. Furthermore, our proposed framework allows for a variety of financial incentives and their required metering topologies.

Our QP-based algorithm requires a user-specified weighting matrix, $\mathbf{H}$. We have presented a heuristic approach to the specification of $\mathbf{H}$. Other approaches are possible, and may provide improved customer benefits.

In the context of feed-in tariffs we assessed the customer benefit of QP energy-shifting by using measured load and generation data from 145 residential customers located in an Australian distribution network. In assessing the potential benefit for each of these customers, we observed that most, but not all, customers see operational savings. Customers who are offered incentives to generate more electricity then they consume, with peak load falling outside the peak and shoulder pricing periods, are included in the category of negative operational savings. Further work is needed to more completely characterize suitable financial policies, metering topologies, and battery size with respect to financial benefits of QP energy-shifting for customers who observed negative operational savings.

\section{Acknowledgments}

Elizabeth L. Ratnam acknowledges the financial support of an Australian Postgraduate Award (APA) and a CSIRO Energy Technology Postgraduate Research Scholarship (Ref: 2011094123).

\section{References}

[1] A. Radchik, I. Skryabin, J. Maisano, A. Novikov, T. Gazarian, Ensuring long term investment for large scale solar power stations: Hedging instruments for green power, Solar Energy 98, Part B (2013) 167-179.

[2] CSIRO, Solar intermittency: Australia's clean energy challenge, Tech. rep., CSIRO (June 2012).

[3] T. E. Del Carpio-Huayllas, D. S. Ramos, R. L. Vasquez-Arnez, Feed-in and net metering tariffs: An assessment for their application on microgrid systems, in: Proc. 6th IEEE/PES Latin America Conf. and Exposition on Transmission and Distribution (T\&D-LA'12), Montevideo, Uruguay, 2012, pp. 1-6.

[4] A. Campoccia, L. Dusonchet, E. Telaretti, G. Zizzo, Feed-in tariffs for grid-connected PV systems: The situation in the European community, in: Proc. IEEE Conf. on Power Tech, Lausanne, Switzerland, 2007, pp. 1981-1986.

[5] L. Hirth, F. Ueckerdt, Redistribution effects of energy and climate policy: The electricity market, Energy Policy 62 (2013) 934-947.

[6] J. von Appen, M. Braun, T. Stetz, K. Diwold, D. Geibel, Time in the sun: the challenge of high PV penetration in the German electric grid, IEEE Power and Energy Mag. 11 (2) (2013) 55-64.

[7] K. Ogimoto, I. Kaizuka, Y. Ueda, T. Oozeki, A good fit: Japan's solar power program and prospects for the new power system, IEEE Power and Energy Mag. 11 (2) (2013) 65-74.

[8] M. J. N. van Werven, M. J. J. Scheepers, The changing role of distribution system operators in liberalised and decentralising electricity markets, in: Proc. IEEE Int. Conf. on Future Power Systems, Amsterdam, Netherlands, 2005, pp. 1-6.

[9] T. Stetz, F. Marten, M. Braun, Improved low voltage grid-integration of photovoltaic systems in Germany, IEEE Trans. Sustain. Energy 4 (2) (2013) 534-542.

[10] R. Tzartzev, W. M. Grady, J. Patel, Impact of high-penetration PV on distribution feeders, in: Proc. 3rd IEEE PES Int. Conf. and Exhibition on Innovative Smart Grid Technologies (ISGT Europe), Berlin, Germany, 2012, pp. 1-6.

[11] M. E. Baran, H. Hooshyar, Z. Shen, A. Huang, Accommodating high PV penetration on distribution feeders, IEEE Trans. Smart Grid 3 (2) (2012) 1039-1046.

[12] R. Tonkoski, D. Turcotte, T. H. M. El-Fouly, Impact of high PV penetration on voltage profiles in residential neighborhoods, IEEE Trans. Sustain. Energy 3 (3) (2012) 518-527.

[13] C. L. Masters, Voltage rise the big issue when connecting embedded generation to long $11 \mathrm{kV}$ overhead lines, Power Engineering Journal 16 (1) (2002) 5-12.

[14] F. Katiraei, J. R. Agüero, Solar PV integration challenges, IEEE Power and Energy Mag. 9 (3) (2011) 62-71. 
[15] C. A. Hill, M. C. Such, D. Chen, J. Gonzalez, W. M. Grady, Battery energy storage for enabling integration of distributed solar power generation, IEEE Trans. Smart Grid 3 (2) (2012) 850-857.

[16] S. Nykamp, A. Molderink, J. L. Hurink, G. J. M. Smit, Storage operation for peak shaving of distributed PV and wind generation, in: Proc. 2013 IEEE PES Conf. on Innovative Smart Grid Technologies (ISGT'13), Washington, DC, 2013, pp. 1-6.

[17] Y. Ueda, K. Kurokawa, T. Tanabe, K. Kitamura, H. Sugihara, Analysis results of output power loss due to the grid voltage rise in gridconnected photovoltaic power generation systems, IEEE Trans. Ind. Electron. 55 (7) (2008) 2744-2751.

[18] R. Tonkoski, L. A. C. Lopes, T. H. M. El-Fouly, Coordinated active power curtailment of grid connected PV inverters for overvoltage prevention, IEEE Trans. Sustain. Energy 2 (2) (2011) 139-147.

[19] H. Farhangi, The path of the smart grid, IEEE Power and Energy Mag. 8 (1) (2010) 18-28.

[20] M. J. E. Alam, K. M. Muttaqi, D. Sutanto, Mitigation of rooftop solar PV impacts and evening peak support by managing available capacity of distributed energy storage systems, IEEE Trans. Power Syst. 28 (4) (2013) 3874-3884.

[21] M. Erol-Kantarci, H. T. Mouftah, Wireless sensor networks for cost-efficient residential energy management in the smart grid, IEEE Trans. Smart Grid 2 (2) (2011) 314-325.

[22] O. Corradi, H. Ochsenfeld, H. Madsen, P. Pinson, Controlling electricity consumption by forecasting its response to varying prices, IEEE Trans. Power Syst. 28 (1) (2013) 421-429.

[23] E. Vrettos, K. Lai, F. Oldewurtel, G. Andersson, Predictive control of buildings for demand response with dynamic day-ahead and real-time prices, in: Proc. 2013 IEEE European Control Conference, Zürich, Switzerland, 2013, pp. 2527-2534.

[24] S. Shao, M. Pipattanasomporn, S. Rahman, Demand response as a load shaping tool in an intelligent grid with electric vehicles, IEEE Trans. Smart Grid 2 (4) (2011) 624-631.

[25] S. Bashash, H. K. Fathy, Modeling and control of aggregate air conditioning loads for robust renewable power management, IEEE Trans. Control Systems Technology 21 (4) (2013) 1318-1327.

[26] D. S. Callaway, I. A. Hiskens, Achieving controllability of electric loads, Proceedings of the IEEE 99 (1) (2011) $184-199$.

[27] M. Pipattanasomporn, M. Kuzlu, S. Rahman, An algorithm for intelligent home energy management and demand response analysis, IEEE Trans. Smart Grid 3 (4) (2012) 2166-2173.

[28] E. Y. Bitar, K. Poolla, Selling wind power in electricity markets: The status today, the opportunities tomorrow, in: Proc. American Control Conference (ACC'12), Montreal, QC, 2012, pp. 3144-3147.

[29] C. Ibars, M. Navarro, L. Giupponi, Distributed demand management in smart grid with a congestion game, in: Proc. 1st IEEE Int. Conf. on Smart Grid Communications (SmartGridComm'10), Gaithersburg, MD, 2010, pp. 495-500.

[30] A.-H. Mohsenian-Rad, A. Leon-Garcia, Optimal residential load control with price prediction in real-time electricity pricing environments, IEEE Trans. Smart Grid 1 (2) (2010) 120-133.

[31] S. Nykamp, M. G. C. Bosman, A. Molderink, J. L. Hurink, G. J. M. Smit, Value of storage in distribution grids - competition or cooperation of stakeholders?, IEEE Trans. Smart Grid 4 (3) (2013) 1361-1370.

[32] P. Palensky, D. Dietrich, Demand side management: Demand response, intelligent energy systems, and smart loads, IEEE Trans. Ind. Informatics 7 (3) (2011) 381-388.

[33] A. Nottrott, J. Kleissl, B. Washom, Energy dispatch schedule optimization and cost benefit analysis for grid-connected, photovoltaic-battery storage systems, Renewable Energy 55 (2013) 230 - 240.

[34] Y. Ru, J. Kleissl, S. Martínez, Storage size determination for grid-connected photovoltaic systems, IEEE Trans. Sustain. Energy 4 (1) (2013) $68-81$.

[35] Y. Ru, J. Kleissl, S. Martínez, Exact sizing of battery capacity for photovoltaic systems, European Journal of Control 20 (1) (2014) $24-37$.

[36] Y. Riffonneau, S. Bacha, F. Barruel, S. Ploix, Optimal power flow management for grid connected PV systems with batteries, IEEE Trans. Sustain. Energy 2 (3) (2011) 309-320.

[37] T. Hubert, S. Grijalva, Modeling for residential electricity optimization in dynamic pricing environments, IEEE Trans. Smart Grid 3 (4) (2012) 2224-2231.

[38] N. R. Darghouth, G. Barbose, R. H. Wiser, Customer-economics of residential photovoltaic systems (Part 1): The impact of high renewable energy penetrations on electricity bill savings with net metering, Energy Policy 67 (2014) $290-300$.

[39] A. J. Black, Financial payback on California residential solar electric systems, Solar Energy 77 (4) (2004) 381 - 388.

[40] B. Sweet, California's energy-storage mandate, Tech. rep., IEEE Spectrum EnergyWise Newsletter (6th Nov. 2013).

[41] C. Candelise, M. Winskel, R. J. K. Gross, The dynamics of solar PV costs and prices as a challenge for technology forecasting, Renewable \& Sustainable Energy Reviews 26 (2013) 96-107.

[42] N.-K. C. Nair, N. Garimella, Battery energy storage systems: Assessment for small-scale renewable energy integration, Energy \& Buildings 42 (11) (2010) 2124-2130.

[43] S. Brown, D. Pyke, P. Steenhof, Electric vehicles: The role and importance of standards in an emerging market, Energy Policy 38 (7) (2010) $3797-3806$.

[44] E. L. Ratnam, S. R. Weller, C. M. Kellett, An optimization-based approach for assessing the benefits of residential battery storage in conjunction with solar PV, in: Proc. IX IEEE Int. Symp. Bulk Power System Dynamics and Control (IREP'13), Rethymno, Greece, 2013, pp. $1-8$.

[45] N. C. Nair, R. Nayagam, R. Francis, New Zealand utility experiences with demand side management, in: Proc. 2008 IEEE Power \& Energy Society General Meeting, Pittsburgh, PA, 2008, pp. 1-5.

[46] K. Sedghisigarchi, Residential solar systems: Technology, net-metering, and financial payback, in: Proc. IEEE Conf. on Electrical Power \& Energy (EPEC), Montreal, Canada, 2009, pp. 1-6. 